IIT-HEP-01/1

hep-ex/0101024

\title{
Prospects for low-energy antiproton physics at Fermilab*
}

Daniel M. Kaplan ${ }^{\dagger a}$

allinois Institute of Technology,

Chicago, Illinois 60616, USA

Fermilab has long had the world's most intense antiproton source, but the opportunities for medium-energy antiproton physics have been limited, and those for low-energy antiproton physics nonexistent. The conclusion of E835 brings this era to an end. While the future of antiproton physics at Fermilab remains highly uncertain, developments are occurring that may lead to a low-energy program within the next several years, with the possibility of an improved medium-energy program thereafter. These issues were considered at the recent $\bar{p} 2000$ Workshop at Illinois Institute of Technology. I summarize the current status of the Fermilab antiproton facility, review hyperon $C P$ violation as an example of the physics that might be achievable, and discuss future possibilities.

\section{1. $\bar{p} 2000$ WORKSHOP: FUTURE OF $\bar{p}$ S AT FERMILAB}

The $\bar{p} 2000$ Workshop was held Aug. 3-5, 2000 at Illinois Institute of Technology. Its goal was to explore the physics potential of a possible new low-energy antiproton storage ring at Fermilab and begin the process of planning for its construction and exploitation. Thirty-nine physicists attended from Europe, Asia, and the U.S., and promising ideas were presented. The major questions addressed could be summarized as follows:

1. What will be Fermilab's capabilities in $\bar{p}$ physics during the next several years?

2. Can a strong physics program be identified to take advantage of these capabilites?

\subsection{Physics motivations}

In particular, we considered two motivating ideas and four general physics areas in which significant progress might be made in a program at Fermilab:

- Fermilab is now starting to define its directions for the post-LHC era. Further broadening of the non- "energy frontier" physics program (beyond BTeV and the neutrino and Main Injector fixed-target efforts) could be an appealing option if a strong clientele can be found.

\footnotetext{
*To appear in Proceedings of the LEAP 2000 Conference, Venice, Italy, Aug. 20-26, 2000.

†E-mail: kaplan@fnal.gov
} 
- Fermilab already has the world's highest-intensity antiproton source, and the available $\bar{p}$ intensity will continue to increase.

- The physics reach in $\bar{p} p \rightarrow$ hyperons might benefit from greater luminosity.

- The physics reach in $\bar{p} p \rightarrow$ charmonium might benefit from greater luminosity, higher energy, and improved energy precision.

- The physics reach in $\bar{p} p \rightarrow$ glueballs or gluonic hybrids might benefit from more running and higher energy.

- A facility for decelerating and trapping antiprotons at Fermilab could provide much more beam than the AD.

\subsection{Current capabilities}

The $\bar{p}$ source now produces cooled antiprotons at a maximum "stacking rate" of $\approx 10 \mathrm{~mA} / \mathrm{hr}$. Given the circumference of the antiproton accumulator, this corresponds to a production rate of $10^{11}$ antiprotons/hr and can support a maximum luminosity of about $2 \times 10^{32} \mathrm{~cm}^{-2} \mathrm{~s}^{-1}$, i.e. beyond this luminosity collisions would consume antiprotons faster than they are produced. The goals for Tevatron Run II are $20 \mathrm{~mA} / \mathrm{hr}$ by March, 2001, and $100 \mathrm{~mA} / \mathrm{hr}$ by Run IIB $(\approx 2005)$.

The antiproton fixed-target run of Experiment 835 (charmonium production) ends in November, 2000, after which no further experimental running in the accumulator is expected. Once Run II begins, the accumulator is expected to be fully dedicated to supplying antiprotons for the Tevatron Collider, in support of two (and, once BTeV is running, possibly three) $2 \mathrm{TeV}$ collision regions operating simultaneously at $2 \times 10^{32} \mathrm{~cm}^{-2} \mathrm{~s}^{-1}$. Thus once Run II begins, Fermilab's approved and existing capabilities in low-energy antiproton physics will be nil.

\subsection{Possible low-energy- $\bar{p}$ upgrade}

In recent months Fermilab's G. Jackson has been exploring the possibility of building a new, small, low-energy $\bar{p}$ facility at Fermilab. The immediate motivations for such a facility come from fields outside nuclear and particle physics [1,2]: NASA is pursuing ideas for $\bar{p} p$-annihilation-fueled interstellar travel in the long-term future and is interested in a source of trapped antiprotons to begin investigating them. Ideas have also been discussed for possible medical applications of trapped antiprotons: production of short-lived isotopes for positron-emission tomography and use of antiprotons for cancer therapy. Exploratory efforts to investigate provision of trapped antiprotons for these purposes are going ahead, subject to the boundary condition that they remain parasitic to commissioning and highenergy running of the Tevatron Collider and that they consume at most a few percent of the available antiprotons.

As discussed below, a stacking rate consistent with $10^{33} \mathrm{~cm}^{-2} \mathrm{~s}^{-1}$ luminosity may be a desirable capability for a future facility; at least it is a plausible upper limit. Such a high stacking rate is quite conceivable but may require upgrading the proton source. It has been estimated [3] that the proposed "Proton Driver" upgrade [4] will increase the intensity in the Main Injector by a factor $\approx 4$. If other bottlenecks in the $\bar{p}$ production process can be overcome, this will translate into a similar factor in stacking rate. 


\section{A PHYSICS EXAMPLE}

To understand some of the issues for a future low-energy antiproton facility (in particular, the need for $10^{33}$ luminosity), we consider in some detail a challenging physics example: hyperon $C P$ violation.

\subsection{Hyperon $C P$ violation}

In addition to $C P$ violation in kaon decays [5], the Standard Model predicts a slight $C P$ asymmetry in decays of hyperons [6 8]. The most accessible signals involve comparison of the (nonuniform) angular distributions of the decay products of polarized hyperons with those of the corresponding antihyperons [7]. For a precision measurement, it is necessary to know the polarizations of the initial hyperons and antihyperons to high accuracy.

By angular-momentum conservation, in the decay of a spin- $1 / 2$ hyperon to a spin- $1 / 2$ baryon plus a pion, the final state must be either S-wave or P-wave. As is well known, the interference term between the $\mathrm{S}$ - and $\mathrm{P}$-wave decay amplitudes gives rise to parity violation, parametrized by Lee and Yang [9] in terms of two independent parameters $\alpha$ and $\beta$ : $\alpha$ is proportional to the real and $\beta$ to the imaginary part of this interference term. $C P$ violation can be sought as a difference in $|\alpha|$ or $|\beta|$ for a hyperon decay and its $C P$-conjugate antihyperon decay or as a particle-antiparticle difference in the partial widths for such decays [7, 10].

Table 11 summarizes the experimental situation. The first three experiments cited studied $\Lambda$ decay only [11]13], setting limits on the $C P$-asymmetry parameter [7,10]

$$
A_{\Lambda} \equiv \frac{\alpha_{\Lambda}+\alpha_{\bar{\Lambda}}}{\alpha_{\Lambda}-\alpha_{\bar{\Lambda}}}
$$

where $\alpha_{\Lambda}\left(\alpha_{\bar{\Lambda}}\right)$ characterizes the $\Lambda(\bar{\Lambda})$ decay to (anti)proton plus charged pion and, if $C P$ is a good symmetry in hyperon decay, $\alpha_{\Lambda}=-\alpha_{\bar{\Lambda}}$.

Fermilab E756 [14 and CLEO [15] employed a new technique in which the cascade decay of charged $\Xi$ hyperons is used to produce polarized $\Lambda$ s, in whose subsequent decay the slope of the (anti)proton angular distribution in the "helicity" frame measures the product of $\alpha_{\Xi}$ and $\alpha_{\Lambda}$. If $C P$ is a good symmetry in hyperon decay this product should be identical for $\Xi$ and $\bar{\Xi}$ events. The $C P$-asymmetry parameter measured is thus

$$
A_{\Xi \Lambda} \equiv \frac{\alpha_{\Xi} \alpha_{\Lambda}-\alpha_{\Xi} \alpha_{\bar{\Lambda}}}{\alpha_{\Xi} \alpha_{\Lambda}+\alpha_{\bar{\Xi}} \alpha_{\bar{\Lambda}}} \approx A_{\Xi}+A_{\Lambda}
$$

The power of this technique derives from the large $\alpha$ value for the $\Xi \rightarrow \Lambda \pi$ decay $(\alpha=0.64)$. A further advantage in the fixed-target case is that within a given $\left.{ }^{(} \bar{\Xi}\right)$ momentum bin the acceptances and efficiencies for $\Xi$ and $\Xi$ decays are very similar, since the switch from detecting $\Xi$ to detecting $\bar{\Xi}$ is made by reversing the polarities of the magnets, making the spatial distributions of decay products across the detector apertures almost identical for $\Xi$ and for $\bar{\Xi}$. (There are still residual systematic uncertainties arising from the differing cross sections for $p$ and $\bar{p}$ and for $\pi^{+}$and $\pi^{-}$to interact in the material of the spectrometer.)

Subsequent to E756, this technique has been used in the "HyperCP" experiment [16] (Fermilab E871), depicted schematically in Fig. 1, which ran during 1996-99. Like E756, HyperCP used a secondary charged beam produced by primary protons interacting in a metal target. The secondary beam was momentum- and sign-selected by means of a curved 

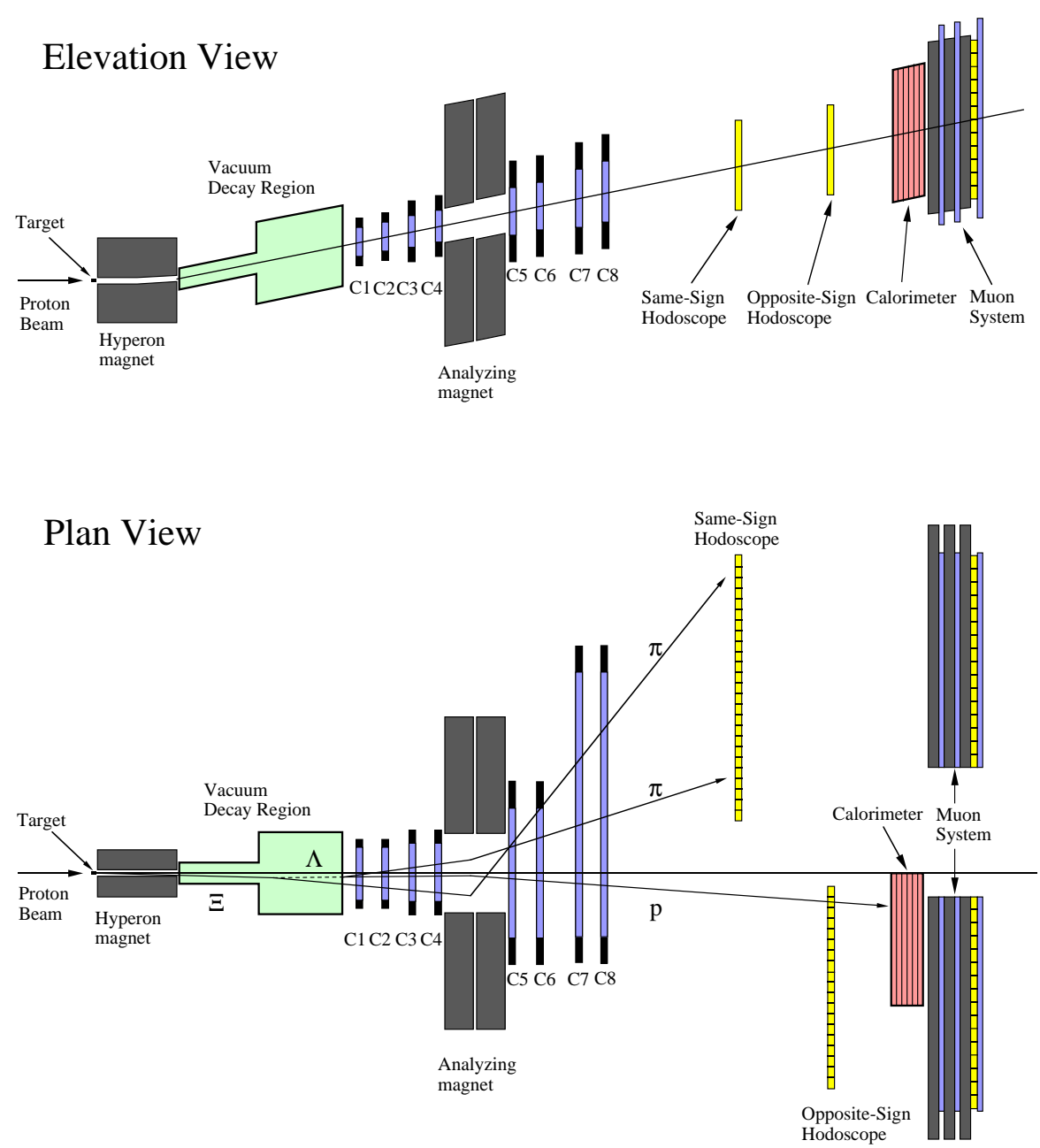

Figure 1. Schematic of the HyperCP spectrometer, comprising eight (or in the 1999 run, nine) MWPC stations (C1-C8) located downstream of the hyperon channel and decay pipe and surrounding an analyzing magnet. The trigger calorimeter and hodoscopes are located far downstream of the analyzing magnet, where the hyperon decay products have separated from each other and from the beam, allowing the trigger elements to be kept outside the $\approx 20 \mathrm{MHz}$ charged secondary beam. The muon detectors give sensitivity to rare decays.

collimator located within a 6-m-long dipole magnet. No measurements were made until after the 13-m-long (evacuated) decay region. HyperCP recorded the world's largest samples of decays of the $\Xi^{-}$and $\bar{\Xi}^{+}$, amounting to $2 \times 10^{9}$ and $0.5 \times 10^{9}$ events, respectively. When the analysis is complete, these should determine $A_{\Xi \Lambda}$ with a statistical uncertainty

$$
\delta A=\frac{1}{2 \alpha_{\Xi} \alpha_{\Lambda}} \sqrt{\frac{3}{N_{\Xi^{-}}}+\frac{3}{N_{\Xi^{+}}}}=1.4 \times 10^{-4} .
$$

The Standard Model predicts this asymmetry to be of order $10^{-5}[7]$. Thus if HyperCP sees a significant effect, it will be evidence for $C P$ violation in the baryon sector substantially larger than predicted by the Standard Model. 
Table 1

Summary of experimental limits on $C P$ violation in hyperon decay.

\begin{tabular}{lccc}
\hline Experiment & Facility & Mode & $\left.A_{\Lambda}{ }^{*}\right]$ or $A_{\Xi \Lambda}\left[^{\dagger}\right]$ \\
\hline \hline R608 & ISR & $p p \rightarrow \Lambda X, p p \rightarrow \bar{\Lambda} X$ & $-0.02 \pm 0.14^{*}$ \\
DM2 & Orsay & $e^{+} e^{-} \rightarrow J / \psi \rightarrow \Lambda \bar{\Lambda}$ & $0.01 \pm 0.10^{*}$ \\
PS185 & LEAR & $\bar{p} p \rightarrow \bar{\Lambda} \Lambda$ & $0.006 \pm 0.015^{*}$ \\
E756 & Fermilab & $p N \rightarrow \Xi^{-} X, \Xi^{-} \rightarrow \Lambda \pi^{-}$, & $0.012 \pm 0.014^{\dagger}$ \\
& & $p N \rightarrow \bar{\Xi}^{+} X, \bar{\Xi}^{+} \rightarrow \bar{\Lambda} \pi^{+}$ & \\
\multirow{2}{*}{ CLEO } & CESR & $e^{+} e^{-} \rightarrow \Xi^{-} X, \Xi^{-} \rightarrow \Lambda \pi^{-}$, & $-0.057 \pm 0.064 \pm 0.039^{\dagger}$ \\
& & $e^{+} e^{-} \rightarrow \bar{\Xi}^{+} X, \bar{\Xi}^{+} \rightarrow \bar{\Lambda} \pi^{+}$ & \\
\hline
\end{tabular}

\subsection{A future experiment}

Whether or not HyperCP observes a statistically-significant effect, it is of interest to ask whether an experiment with substantially larger event samples is feasible [17]. Since HyperCP sensitivity is an order of magnitude short of the Standard Model prediction, a desirable goal would be two orders of magnitude in sample size.

We have begun to explore this question. While we believe that the approach taken in HyperCP is near the limit of what is possible with present-day particle-detection technology, ${ }^{3}$ an alternative approach pioneered by the PS185 Collaboration at CERN may have the requisite "head room." The PS185 experiment [18] operated at the Low-Energy Antiproton Ring (LEAR) at CERN between 1984 and 1996 and utilized $\bar{p} p$ annihilation slightly above the threshold for production of a $\bar{\Lambda} \Lambda$ pair. (In this case, the requirement that the hyperon and antihyperon polarizations be precisely known is modified, since by $C$-parity conservation in the strong interaction the polarizations of the hyperons and antihyperons are equal.(1)

Limited by the available antiproton intensity at LEAR, PS185 has achieved a sensitivity of only $1.5 \%$ 13. However, in the early 1990s the CERN "CP-Hyperon Study Group" designed a hyperon $C P$-violation experiment for SuperLEAR aimed at $10^{-4}$ sensitivity [21] (see Fig. 2). While SuperLEAR was never built, the antiproton production rate at the Antiproton Source at Fermilab is already at least four orders of magnitude beyond that achieved at LEAR, and, as mentioned above, substantial improvements to its capabilities are planned. A new antiproton storage ring at Fermilab capable of producing $\bar{\Lambda} \Lambda$ events at a $60 \mathrm{kHz}$ rate may be feasible at relatively modest cost [1]. This would allow the accumulation of a sample of order $10^{11}$ good events within a few years' running time [17]. Challenges that will need to be met include the design of beam optics and a gas-jet target that permit $\approx 10^{33} \mathrm{~cm}^{-2} \mathrm{~s}^{-1}$ luminosity, detecting the $\Lambda$ decay products and reconstructing their tracks with good efficiency at $\approx 200 \mathrm{MHz}$ charged-particle rate, triggering with good efficiency and adequate background rejection at $\approx 100 \mathrm{MHz}$ interaction rate, and acquiring data at the

\footnotetext{
${ }^{3}$ The high rate of secondary beam in HyperCP — about $20 \mathrm{MHz}$ spread over an area of several $\mathrm{cm}^{2}-$ caused detector inefficiencies in the beam region at the percent level (in the most upstream MWPCs) due to MWPC deadtime.

${ }^{4}$ This assumption is in fact not experimentally tested at the level required for this measurement, but the limit $B\left(\pi^{0} \rightarrow 3 \gamma\right)<3.1 \times 10^{-8}$ [19] suggests that it is a good one [20]; in any case, $C$ violation in the strong production process would be as interesting a discovery as $C P$ violation in the weak decay [21].
} 


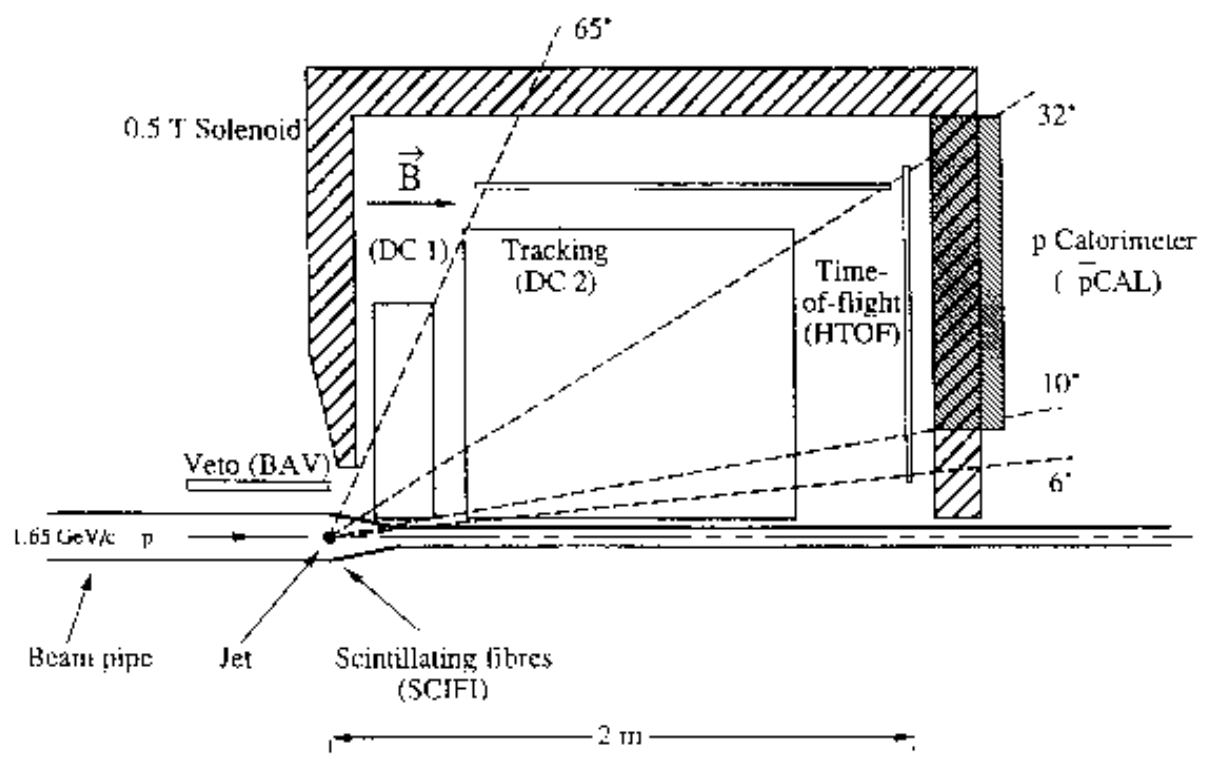

Figure 2. Schematic of $\bar{\Lambda} \Lambda$ spectrometer designed for SuperLEAR (from Ref. [21]).

resulting high trigger rate [22].

\subsection{Additional physics}

Beyond hyperon $C P$ violation, a low-energy $(p \approx 2 \mathrm{GeV} / c)$, high-intensity antiproton storage ring equipped with a high-luminosity fixed-target spectrometer may be able to carry out experiments designed to study rare hyperon decays, hyperon beta decays, quark confinement, and soft QCD effects [23]. A "universal" fixed-target $\bar{p} p$ spectrometer is under study [24]. Studies of charmonium and possible gluonic-hybrid charmonium states would require higher antiproton energies $(p \lesssim 10 \mathrm{GeV} / c)$. Experiments in this energy regime could address several open issues related to charmonium and QCD, including the relative $J / \psi$ and $\psi^{\prime}$ decay widths, $\chi_{c}$ widths, masses of the charmonium pseudoscalar states, the value of the strong coupling constant $\alpha_{s}$ at the charm-quark mass, existence and properties of higher radial excitations of charmonium, and the $J / \psi$-nucleon cross section in the kinematic regime relevant to the interpretation of heavy-ion quark-gluon-plasma searches 25]. Additional topics include the possible existence of glueballs or gluonic hybrids in the $>2.5 \mathrm{GeV} / c^{2}$ mass region (where their interpretation may be clearer than at low mass) and precision measurements of the $\tau$ and $\nu_{\tau}$ masses [25]. Experiments with trapped antiprotons include a variety of $C P T$ tests, for example precision studies of the hyperfine structure of antihydrogen and of the gravitational interaction between antihydrogen and the Earth [26,27]. These might best be pursued in an atomic-beam approach rather than at rest, thus a storage ring may have advantages over the pulsed beam provided by the AD [26].

\section{A SCENARIO FOR A NEW FERMILAB FACILITY}

Jackson has outlined a scenario leading to a new low-energy antiproton storage-ring facility at Fermilab [1,28]. The proposed approach is to use the Main Injector to decelerate cooled 
antiprotons from the accumulator down to the injection momentum of a new small storage ring $(\approx 2 \mathrm{GeV} / c)$. While this is below the Main Injector's design momentum range, the ability of the Main Injector magnets and their power supplies to operate stably at this momentum has already been demonstrated, and a suitable operating mode for the RF system has been devised and demonstrated to decelerate a proton beam down to $3 \mathrm{GeV} / c$ [2]. (In the Oct. 2000 test, a software limitation prevented the demonstration of deceleration to $2 \mathrm{GeV} / c$, but this is expected to be rectified soon so that a test of deceleration to $2 \mathrm{GeV} / c$ will become feasible [2].)

If the Main Injector indeed can provide $2 \mathrm{GeV} / c$ antiprotons, the next steps require construction of an extraction system for the decelerated antiprotons followed by a transfer line to a location (say the MI-8 service area) convenient for installation and operation of experimental trapping apparatus. At this stage a low intensity of trapped antiprotons could be provided, using a degrader to lower the momentum sufficiently for trapping.

To provide a higher-quality and higher-intensity beam, the $2 \mathrm{GeV} / c$ ring will be needed. It should be equipped with enough RF to decelerate down to the tens to hundreds of $\mathrm{MeV} / c$ range. With electron cooling in this ring, very high luminosities $\left(\sim 10^{33}\right)$ should be feasible. At this stage, hyperon experiments, etc. (as discussed above) become feasible, provided that sufficient antiproton flux is available to satisfy their needs. Interleaved operation of other lower-flux experiments, including trapped $\bar{p}$ studies using degraders or RFQ deceleration, should be possible with only minor impact on the duty factor of the "major user."

To provide a maximum of flexibility, an additional, larger storage ring could be added to the facility to provide antiprotons over the $\approx 1-10 \mathrm{GeV} / c$ range, allowing both experiments at higher momenta and periodic filling of traps with minimal impact on the high-luminosity rare-hyperon-effect studies. However, whether such a ring would be worth installing at Fermilab would depend on how the GSI upgrade proposal [24,29] fares.

The scenario just presented is being pursued aggressively by a private company, Technanogy, LLC, with funding from NASA, and the small storage ring could be in operation within the next several years. It will soon behoove potential users of the facility to work seriously on the design of the challenging experimental apparatus that will be required, as well as to specify needed machine capabilities so that these may be folded into the design process.

\section{Acknowledgements}

I thank the organizers for inviting me to speak at this stimulating conference, convened at so striking a location. This work was supported in part by grants from the Illinois Board of Higher Education, the Illinois Department of Commerce and Community Affairs, and the U.S. Department of Energy.

\section{REFERENCES}

1. G. P. Jackson, "Accelerator Possibilities for Low Energy Antiprotons at the Fermi National Accelerator Laboratory," in Proc. $\bar{p} 2000$ Workshop, D. M. Kaplan and H. A. Rubin, eds., Illinois Institute of Technology, Chicago, IL 60616, USA, Aug. 3-5, 2000.

2. G. P. Jackson, private communication.

3. W. Chou, in Proc. $\bar{p} 2000$ Workshop, op cit. 
4. S. D. Holmes, Fermilab Report Fermilab-TM-2021 (1997); W. Chou, Proc. 1999 Particle Accelerator Conference, A. Luccio and W. MacKay, eds. (IEEE, New York, 1999), p. 3285.

5. See for example J. L. Rosner, in Proc. 8th Jorge Andre Swieca Summer School: Particles and Fields, Rio de Janeiro, Brazil, 5-18 Feb. 1995, J. Barcelos-Neto, S. F. Novaes, V. O. Rivelles, eds., World Scientific (1996), p. 116.

6. A. Pais, Phys. Rev. Lett. 3, 242 (1959); O. E. Overseth and S. Pakvasa, Phys. Rev. 184, 1663 (1969); J. F. Donoghue and S. Pakvasa, Phys. Rev. Lett. 55, 162 (1985).

7. J. F. Donoghue, X.-G. He, S. Pakvasa, Phys. Rev. D 34, 833 (1986); X.-G. He, H. Steger, G. Valencia, Phys. Lett. B 272 (1991) 411.

8. G. Valencia, Proc. $\bar{p} 2000$ Workshop, op cit.

9. T. D. Lee and C. N. Yang, Phys. Rev. 108, 1645 (1957).

10. J. F. Donoghue, B. R. Holstein, and G. Valencia, Phys. Lett. 178B, 319 (1986) and Int. J. Mod. Phys. A 2, 319 (1987).

11. P. Chauvat et al., Phys. Lett. 163B (1985) 273.

12. M. H. Tixier et al., Phys. Lett. B212 (1988) 523.

13. P. D. Barnes et al., Nucl. Phys. B (Proc. Suppl.) 56A (1997) 46.

14. K. B. Luk et al., "Search for Direct CP Violation in Nonleptonic Decays of Charged Xi and Lambda Hyperons," hep-ex/0007030 (2000).

15. D. E. Jaffe et al., "Search for Direct CP Violation in $\Xi$ Hyperon Decay," CLNS 98/1587, CLEO 98-16 (2000).

16. J. Antos et al., Fermilab Proposal 871 (revised), Mar. 26, 1994; see also http://ppd.fnal.gov/experiments/e871/welcome.htm.

17. D. M. Kaplan, in Proc. Hyperon 99: Hyperon Physics Symposium, Batavia, Illinois, 27-29 Sept. 1999, D. A. Jensen, E. Monnier, eds., Fermilab-Conf-00/059, p. 165.

18. P. Barnes et al., Nucl. Phys. A526, 575 (1991).

19. J. M. McDonough et al., Phys. Rev. D 38, 2121 (1988).

20. S. Pakvasa, private communication.

21. N. Hamann et al., CERN/SPSLC 92019, SPSLC/M491, 30 March 1992.

22. Further discussion of experimental issues for a high-sensitivity hyperon- $C P$-violation experiment may be found in D. M. Kaplan, "Towards a Hyperon "Super- $C P$ " Experiment: Report of the Hyperon-CP-Violation Working Group," Proc. $\bar{p} 2000$ Workshop, op cit.

23. See various articles in Proc. $\bar{p} 2000$ Workshop, op cit.

24. See the GSI upgrade Letter of Intent at http://tau.ep1.ruhr-uni-bochum.de/gsi/,

25. K. K. Seth, "Challenges and Opportunities in Antiproton Physics in the Charmonium Region," in Proc. $\bar{p} 2000$ Workshop, op cit.

26. M. H. Holzscheiter, "Physics with Ultra-Low Energy Antiprotons," in Proc. $\bar{p} 2000$ Workshop, op cit.

27. T. J. Phillips, "An Antihydrogen Interferometer for Measuring Antimatter Gravity," in Proc. $\bar{p} 2000$ Workshop, op cit.

28. See http://hbar.fnal.gov/.

29. W. Henning, "Antiprotons at the Future GSI Gacility," in Proc. $\bar{p} 2000$ Workshop, op cit.; V. Metag, this Conference. 